\title{
Impacts des banquettes dans la récupération des terres dégradées au Niger
}

\author{
LAMINOU MANZO Ousmane ${ }^{1 *}$, AMANI Abdou², DAN GUIMBO Iro33, RACHIDI Abdou Harouna ${ }^{4}$, \\ MAHAMANE Ali5 \\ 'Département de Génie Rural et Eaux \& Forêts, Faculté d'Agronomie et des Sciences de l'Environnement, Université \\ Dan Dicko Dankoulodo de Maradi, B.P. 465 Maradi, Niger \\ 2Département de Gestion des Ressources Naturelles, Institut National de la Recherche Agronomique du Niger \\ (INRAN), BP 429 Niamey, Niger \\ 32Departement de Génie Rural et Eaux \& Forêts, Faculté d’Agronomie, Université Abou Moumouni de Niamey, B.P. \\ 10960 Niamey, Niger \\ 4Direction Générale des Eaux et Forêts, Ministère de l'Environnement et du Développement durable, Niamey, Niger \\ ${ }^{5}$ Département de Biologie, Faculté des Science et Technique, Université Abou Moumouni de Niamey, B.P. 10960 \\ Niamey, Niger
}

*Auteur correspondant : Email : lamine_ous@yahoo.fr - Tél : +22796493560

Original submitted in on $8^{\text {th }}$ May 2020. Published online at www.m.elewa.org/journals/ on $31^{\text {st }}$ July 2020

https://doi.org/10.35759/JABs.151.1

\section{RÉSUMÉ}

Objectifs : Le département de Ouallam au Niger est soumis au phénomène de dégradation des terres. La présente étude, conduite sur le plateau dégradé de Sini Bangou dans la Commune de Simiri, a pour objectif d'évaluer l'efficacité du dispositif des banquettes anti-érosives sur l'amélioration de la couverture végétale et de la qualité du sol.

Méthodologie et résultats : Le dispositif expérimental de collecte des données est un site de récupération des terres où des banquettes ont été réalisées, plantées et ensemencées. La méthodologie a consisté à une analyse diachronique de l'occupation $d u$ sol à l'aide des images Landsat, une analyse granulométrique, des tests d'infiltration de l'eau et des relevés phytosociologiques. Les résultats montrent une amélioration du couvert végétal de 17,24 ha, soit $11,50 \%$ de la superficie du site, l'apparition d'une végétation spontanée de 69 espèces dont 11 ligneuses appartenant à 7 familles et 58 herbacées réparties dans 15 familles dominées par la famille des Poaceae $(31,88 \%)$ et les types biologiques Thérophytes $(69,01 \%)$.Les espèces à distribution Soudano-Zambéziennes $(33,21 \%)$ etSoudano-ZambéziennesSahariennes-Sindiennes(31,15\%) sont les types phytogéographiques les plus dominants de la flore globale à l'échelle africaine. La vitesse de l'infiltration relativement lente enregistrée pendant les dix premières minutes $(\leq 10 \mathrm{~cm} / \mathrm{mn})$, a progressé au rythme d'une moyenne de $1 \mathrm{~cm} / \mathrm{mn}$ en profondeur pour s'arrêter au bout de 30 minutes au profit d'une infiltration latérale. L'épaisseur des dépôts de sédiments varie entre 9 et $15 \mathrm{~cm}$ selon les banquettes et la teneur en matière organique fluctuant entre 0,36 à 1,43\%, quoique faible, reste plus importante au niveau des horizons de surface de $0-20 \mathrm{~cm}$ que ceux de profondeur $20-40 \mathrm{~cm}$. Ces effets associés au caractère dominant sableux du sol ont permis l'amélioration de l'état structural et textural de ce dernier et l'augmentation de l'infiltration et du stockage de l'eau qui aurait résulté au développement quantitatif et qualitatif du couvert végétal. 
Conclusion et application des résultats : Cette étude montre que les banquettes constituent une méthode efficace pour régénérer le capital productif d'un écosystème dégradé. Cependant, la pérennité des services écosystémiques de l'aménagement devrait être garantie par des modes de gestion rationnelle.

Mots clés : Erosion hydrique, récupération, végétation, sol, infiltration, Niger

\section{Impacts of terraces in the degraded land reclamation in Niger ABSTRACT}

Objectives: The department of Ouallam in Niger is subject to land degradation. The study was conducted on the degraded area of Sini Bangou in the district of Simiri with the aim to assess the effectiveness of the anti-erosion terraces system on improving plant cover and soil quality.

Methodology and results: Experimental data collection design was a degraded land site where terraces have been made with tree plantation and herbaceous seeds sown. The methodology consisted of a diachronic analysis of land use using Landsat images, a granulometric analysis, water infiltration tests and phytosociological surveys. Results show an improvement in the plant cover of 17.24 ha, or $11.50 \%$ of the site area, a spontaneous vegetation of 69 species made up of 11 woody ones belonging to 7 families and 58 herbaceous ones distributed into 15 families dominated by the family of Poaceae $(31.88 \%)$ and the biological types of Therophytes (69.01\%). Soudano-Zambezian (33.21\%) and Soudano-ZambezianSaharan-Sindian $(31.15 \%)$ species are the most dominant phytogeographic types of the overall flora on an african scale. The relatively slow infiltration speed recorded during the first ten minutes $(\leq 10 \mathrm{~cm} / \mathrm{min})$, increased at the rate of an average of $1 \mathrm{~cm} / \mathrm{min}$ in depth to stop after 30 minutes in favour of a lateral infiltration. Sediment deposits thickness varied between 9 and $15 \mathrm{~cm}$ depending on the terraces and the organic matter content fluctuating between 0.36 to $1.43 \%$, although low, remains greater at the level of the surface horizons of $0-20 \mathrm{~cm}$ than those of 20-40 cm deep. These effects associated with the dominant sandy character of the soil allowed the structural and textural soil state improvement and the increase in the water infiltration and storage which would have resulted in the quantitative and qualitative development of the plant cover.

Conclusion and application of findings: The study shows that terraces are an effective method for regenerating the productive capital of a degraded ecosystem. However, the sustainability of the ecosystem services of the treated area should be guaranteed by rational management methods.

Keywords: Water erosion , reclamation, vegetation, soil, infiltration, Niger

\section{INTRODUCTION}

Le Niger, à l'instar des autres pays sahéliens, fait face aux aléas naturels caractérisés par des catastrophes naturelles dont les plus courantes sont hydrométéorologiques notamment les sécheresses, les tempêtes de sable, les inondations (SE/CNEDD, 2006). C'est dans ce contexte particulier que s'exercent les principales activités agropastorales par une population majoritairement rurale à plus de $87 \%$. On assiste à un déséquilibre entre les besoins de la population et le potentiel productif qui se dégrade progressivement à travers un appauvrissement croissant du capital terre, la diminution de la jachère, la surexploitation des ressources végétales et l'intensification du processus de désertification (Hamidou, 2015).Les conditions météorologiques sont caractérisées par des pluies insuffisantes, parfois diluviennes, irrégulières et mal réparties dans l'espace et dans le temps, des vents forts et fréquents et une élévation de la température. L'une des conséquences de ces facteurs climatiques est la dégradation des terres qui est aggravée par les activités humaines notamment celles de production agrosylvopastorale et la démographie (Banque Mondiale, 2013). En effet, la population du Niger, est passée de 2300000 habitants en 1950 à 21466 863habitants en 2018 (INS, 2019) avec un 
taux de croissance de 3,9\%, l'un des plus élevés d'Afrique. Cette augmentation rapide de la population a provoqué l'accélération de la pression sur les ressources naturelles. On considérait qu'en 2010 , environ $25 \%$ de cette population vivraient sur des terres agricoles dégradées, soit sur une surface agricole représentant $34,6 \%$ de la superficie du pays (PNUE, 2007 cité par Emely, 2010).On estime que plus de $40 \%$ de la superficie agricole sont dégradées à fortement dégradées, se traduisant par une baisse tendancielle des rendements dans de nombreuses zones agricoles (SP/SDR, 2010). Dans le même temps, la perte annuelle des terres agricoles est d'environ 100000 à 120000 hectares, alors que les efforts de restauration ne dépassent guère 20000 hectares par an (INS, 2012). Aussi, la perte annuelle en superficie des forêts naturelles est estimée à 37 050 hectares à partir de 2000. Ainsi, entre 1990 et 2010, le pays a perdu 741000 hectares de forêts, ce qui représente $38,1 \%$ de son couvert forestier total (FAO, 2010). La dégradation du potentiel productif induite par ces divers facteurs se manifeste principalement par la formation de vastes glacis dénudés et l'encroûtement des sols favorisant l'érosion hydrique, la formation et l'élargissement des talwegs et ravins et la formation des dunes mouvantes à l'origine des phénomènes d'ensablement (ME/SU/DD, 2014).

\section{MATÉRIEL ET MÉTHODES}

Site d'étude: Le site de récupération des terres de Sini Bangou, objet de la présente étude, est situé à 25 kilomètres au sud-ouest du village de Simiri, entre les latitudes $14^{\circ} 04^{\prime} 08,2^{\prime \prime}$ et $14^{\circ} 02^{\prime} 48,9^{\prime \prime}$ Nord et les longitudes $2^{\circ} 00^{\prime} 35,00^{\prime \prime}$ et $2^{\circ} 02^{\prime} 07,5^{\prime \prime}$ Est. C'est un plateau dégradé vaste de plus de 800 ha caractérisé par un sol latéritique et cuirassé avec une pente variant entre 1 et $3 \%$. La végétation est de type steppique composée des Combrétacées clairsemées et un tapis herbacé dominé par les thérophytes et confiné autour des arbustes. Le climat est de type tropical aride, avec des précipitations annuelles moyennes de 250 à 450 $\mathrm{mm}$ irrégulièrement réparties dans le temps et dans
Les conséquences qui en découlent se traduisent par la perte des caractéristiques physicochimiques des sols, la réduction des espaces agrosylvo-pastoraux, la disparition des espèces végétales combinée à l'insuffisance de la régénération naturelle d'où la perte de la biodiversité et de son habitat. C'est ainsi qu'on assiste à la baisse des revenus des ménages et la persistance de l'insécurité alimentaire. Face à cette situation, le pays a expérimenté plusieurs techniques de protection et de récupération des terres agrosylvopastorales, communément appelées techniques de Conservation des Eaux et des Sols/Défense et Restauration des Sols (CES/DRS), notamment celles de dissipation et de captage des eaux afin de favoriser leur infiltration et la reconstitution de la couverture végétale pour ainsi réduire les risques de ruissellement et d'érosion. Ainsi, plusieurs opérations des travaux de CES/DRS ont été réalisées à travers le pays mais avec des résultats très variables d'un site à un autre. C'est dans ce contexte que la présente étude a été conduite dans le but d'investiguer sur les changements environnementaux induits par la technique des banquettes anti-érosives sur le plateau de Sini Bangou dans la commune rurale de Simiri. L'objectif de celle-ci est d'évaluer l'impact de la technique sur le sol et la végétation.

l'espace. II est caractérisé par une période sèche de 8 à 9 mois (CDR, 2016). Les températures varient en moyenne de $18^{\circ} \mathrm{C}$ (en décembre ou en janvier) à $45^{\circ} \mathrm{C}$ (en mars ou avril) au cours de l'année. En général, les températures sont élevées toute l'année, accentuant ainsi les phénomènes d'évapotranspiration intense qui entraîne par conséquent l'assèchement du sol (Faran, 1984 cité par Souley, 2008). Le site d'étude est à vocation sylvo-pastorale. Les principales menaces identifiées sont la dégradation du couvert végétal et du sol, soumis au ruissellement et au ravinement qui provoquent des inondations au niveau des infrastructures en aval (CDR, 2016). 


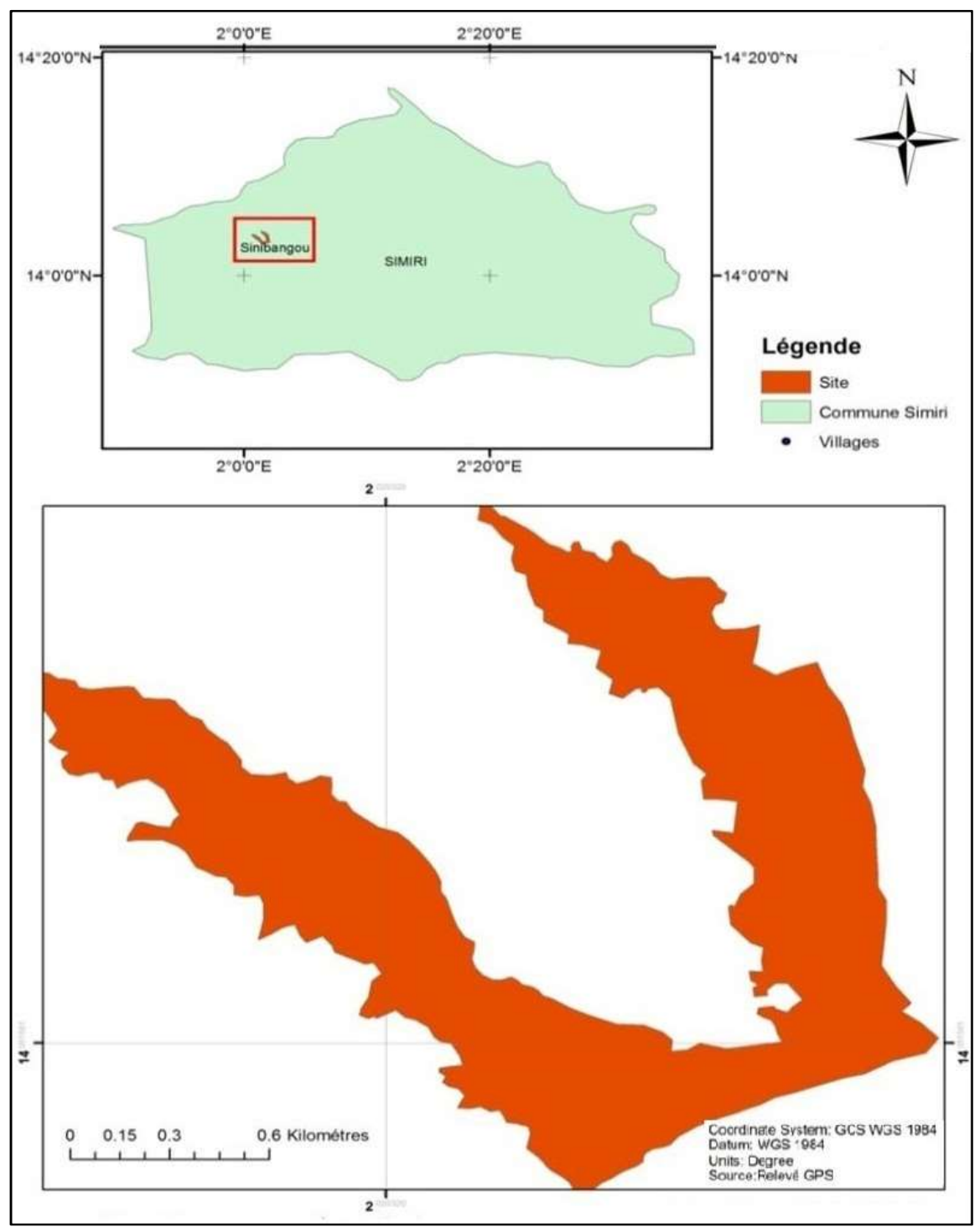

Figure1 : Localisation du site de Sini Bangou

L'aménagement du site (Figure1), réalisé 2015 par le projet PGRC-DU, a consisté à un traitement mécanique, à travers la confection des banquettes, et biologique par la plantation d'arbres et l'ensemencement des herbacées sur une superficie de 150 ha du plateau dégradé entrecoupé par un ravin. La banquette est un ouvrage antiérosif, long de $60 \mathrm{~m}$, réalisé suivant des courbes de niveau. L'ouvrage est ainsi constitué de deux bras latéraux de $10 \mathrm{~m}$ chacun, d'une tranchée large de $2 \mathrm{~m}$ et profonde de 0,20 à 0,40 $\mathrm{m}$ (Photo 1). La densité des ouvrages est de 6 banquettes/ha disposées en quinconce avec un écartement de $15 \mathrm{~m}$ entre elles et de $25 \mathrm{~m}$ entre les lignes, soit un total de 900 banquettes. 

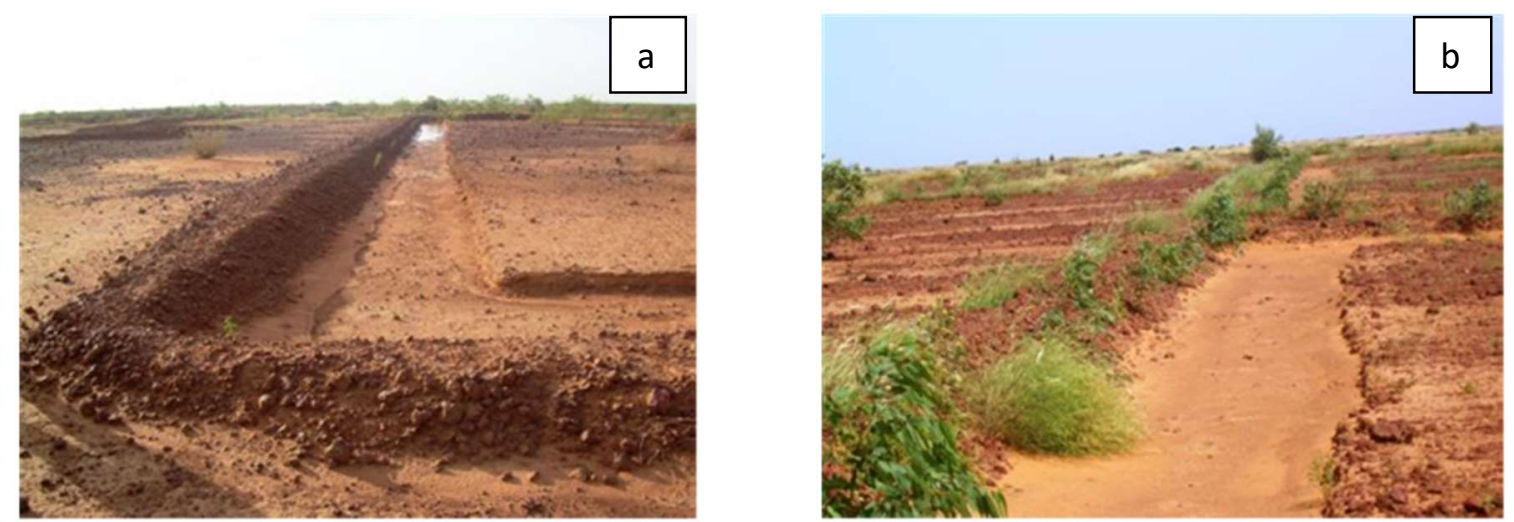

Photo1 : Banquettes réalisées sur le site : Juillet 2015 (a) et Octobre 2015 (b). (Source : PGRC-DU, 2015)

Le traitement biologique réalisé a consisté à une plantation de 18000 plants à raison de 20 plants par banquette, répartis entre les espèces d'Eucalyptus camaldulensis Dehnh et Acacia senegal (L.) Willd. L'ensemencement a été réalisé avec des graines des espèces de Cymbopogon giganteus Chiov., Chenchrus biflorus Roxb. et Pennisetum pedicelatum Trin.

Dispositif de collecte des données : Le dispositif de collecte des données a été déterminé après avoir réalisé un pré-inventaire qui a permis de déterminer le taux de sondage et le nombre total de placettes à prendre en compte. Ainsi, 30 placettes de 0,1 hectare $(50 \mathrm{~m} \times 20 \mathrm{~m})$ équidistantes de $100 \mathrm{~m}$ ont été inventoriées sur deux layons perpendiculaires à l'axe du site. II a été mesuré sur chaque arbre, la hauteur et le diamètre à $1,30 \mathrm{~m}$. A partir de ces données traitées, le taux de sondage et le nombre de placettes à inventorier ont été déterminés. La formule utilisée pour déterminer le nombre placettes est : $N=(t \times C V){ }^{2} / E^{2}$ avec $\mathrm{t}=$ variable de Student ; au seuil de signification $95 \%, t=1,96$ et quand $\mathrm{N}>30$, $\mathrm{t}$ sera arrondi à $2 ; \mathrm{N}=$ Nombre de placettes à inventorier; $E=$ erreur en $\%$ considérée est fixée à $10 \% ; C V=$ coefficient de variation en $\%$, sur la grandeur considérée ici le diamètre.

Le taux de sondage (TS) est calculé à partir de la formule TS $=$ SP $\times$ N/ST avec SP: superficie des placettes ; $N$ : nombre des placettes et ST: superficie totale du site.

L'échantillon de collecte des données est ainsi composé de 69 placettes de $50 \mathrm{~m}$ sur $20 \mathrm{~m}$, distantes entre elles de $100 \mathrm{~m}$ et reparties entre 10 transects parallèles (Figure 2). Ces derniers sont équidistants de $217 \mathrm{~m}$ et largement entrecoupés par un ravin. 


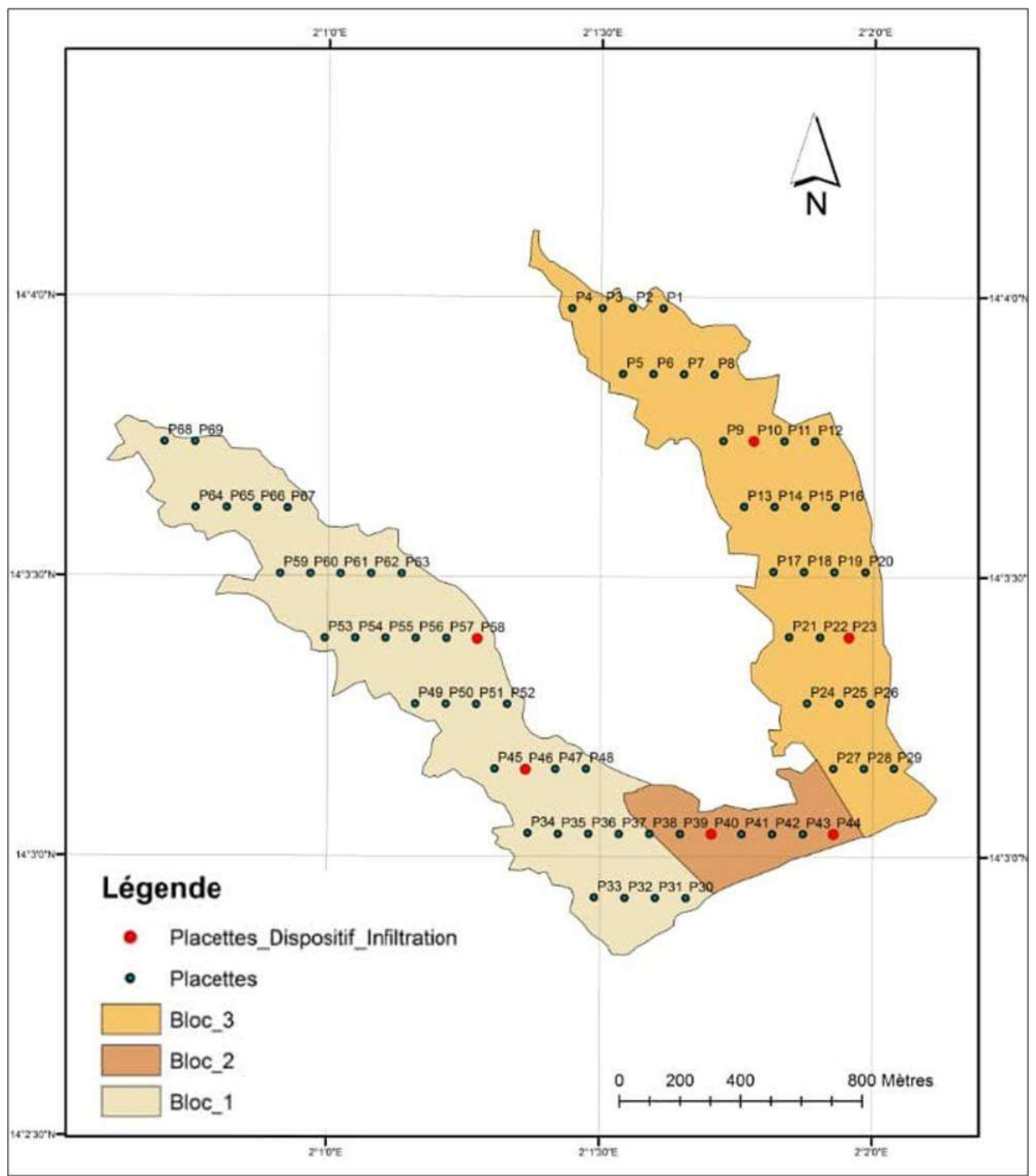

Figure2 : Dispositif de collecte des données

Les placettes sont ainsi disposées sur les trois blocs identifiés du site en tenant compte de la configuration du terrain en forme de ' $U$ '. Le premier bloc est représenté par le côté ouest, le deuxième par la base du 'U' et le troisième par le côté est avec les mêmes caractéristiques que le premier.

Collecte des données: La collecte des données a porté sur l'évolution du couvert végétal, les caractéristiques physico-chimiques du sol notamment la texture et la teneur en matière organique, l'épaisseur des dépôts de sédiments dans les banquettes et le fonctionnement hydrique au niveau des différentes parties la banquette. Pour illustrer l'occupation du sol du plateau par la végétation suite à l'aménagement réalisé, une étude diachronique a été réalisée à partir des images Landsat TM de 2001 et de 2017. La caractérisation du sol a été effectuée à partir des échantillons de sol prélevés dans les banquettes situées dans les placettes de l'échantillon déterminé au niveau des trois entités de la banquette notamment la tranchée, la partie scarifiée et l'impluvium qui fait office de témoin à des profondeurs de $0-20$ et $20-40 \mathrm{~cm}$. Ainsi, un total de dix-huit échantillons a été prélevé au niveau de six banquettes représentatives du site. Dans chaque type de bloc, deux banquettes ont été concernées. Les résultats des blocs 1 et 3 , présentant les mêmes caractéristiques, ont été fusionnés. Après le prélèvement, les échantillons ont été conditionnés dans des emballages plastiques et étiquetés. Pour réaliser un bon diagnostic des risques de ruissellement et d'érosion sur un versant, il convient d'observer le fonctionnement hydrique du terrain au cours de la saison des pluies ou au moins de tester la capacité d'infiltration des sols soumis à diverses utilisations (Roose et al., 1993).Pour caractériser le fonctionnement hydrique du site, le test d'infiltration par 
la méthode conventionnelle des doubles anneaux a été utilisé (Muntz et al.,1905).Les mesures d'infiltration au niveau de la tranchée, des parties scarifiée et non scarifiée de l'impluvium des banquettes. Ainsi, pour chacun des 3 blocs, ces mesures ont été réalisées au niveau 2 banquettes avant les premières pluies afin de minimiser l'impact de la teneur du sol en humidité initiale (Lili et al., 2008). Au total, 18 mesures d'infiltration ont été réalisées. Dans la caractérisation de la végétation, des relevés phytosociologiques ont effectués à l'aide de la méthode sigmatiste de BraunBlanquet (1932) au niveau de 69 placettes que compte l'échantillon. Dans chaque placette, les noms de toutes les espèces végétales ont été enregistrées, la hauteur totale, des diamètres à la base, à $0,20 \mathrm{~m}$ du sol et 1,30 $m$ du sol (DHP), le diamètre du houppier dans les deux directions perpendiculaires des arbres ont été mesurés et leur état sanitaire déterminé. Ces données ont été collectées pour déterminer le taux de survie et l'accroissement moyen annuel (hauteur et diamètre) des espèces plantées, la densité et le recouvrement des ligneux sur le site.

Traitement et analyse des données : La superficie du sol recouverte par la végétation, à la faveur de la réalisation des banquettes du site, a été déterminée grâce au traitement et analyse des images satellitaires à l'aide du logiciel ENVI 4.7.L'image Landsat TM de 2017 a géométriquement été rectifiée et géoréférencée dans le système de projection WGS 84 UTM zone 32. L'image de 2001a été corrigée et géoréférencée dans le même système de référence que celle de 2017. Elles ont été par la suite calibrées, colorées à la composition $5 / 4 / 3$ et traitées à l'aide de l'algorithme du maximum de vraisemblance. Les images classées et validées ont été lissées sous ENVI4.7à l'aide de l'algorithme focal analysis et vectorisées par le même logiciel afin de disposer d'ensembles homogènes d'unités d'occupation des sols et pour leur enregistrement dans un système d'information géographique (SIG). La méthode d'analyse granulométrique des échantillons du sol utilisée a été celle de la pipette de Robinson (Yacouba et al., 2018). Les proportions des classes de diamètre de particules et aussi les classes de textures du sol dans les trois parties des banquettes ont été déterminées à l'aide du graphique triangulaire de Soil Taxonomy de United States Department of Agriculture(USDA) adopté par la FAO. La teneur de la matière organique des échantillons prélevés a consisté à déterminer le carbone organique du sol et la matière organique à l'aide de la méthode de Walkley et Black (1934). Ces analyses des échantillons de sol ont été réalisées au Laboratoire du sol de la Faculté d'Agronomie de l'Université Abdou Moumouni de Niamey. La mesure du dépôt sédimentaire a été réalisée dans les tranchées des banquettes. Après une fouille à l'aide d'une pelle, le dépôt se présente sous forme de stries. Le dépôt correspond à l'accumulation de particules emportées par les agents d'érosion et qui se déposent dans la banquette. L'épaisseur du dépôt a été mesurée à l'aide d'une règle graduée de $50 \mathrm{~cm}$. Le fonctionnement hydrique a été caractérisé à l'aide du test d'infiltration du site par la méthode conventionnelle des doubles anneaux (Muntz et al., 1905). Elle consiste à maintenir une charge d'eau constante dans l'infiltromètre à double anneaux enfoncés dans le sol et dans lesquels le fluide va s'infiltrer. Le test a été réalisé sur les différentes entités de la banquette notamment la tranchée, la partie scarifiée et l'impluvium, considéré comme témoin. La méthode des relevés phytosociologiques de Braun-Blanquet (1932), qui a été utilisée, se base sur une classification qui permet d'estimer visuellement la couverture de chaque espèce sur un site donné en lui attribuant les coefficients d'abondance-dominance de Braun-Blanquet.

L'échelle d'abondance dominance utilisée a été la suivante :

5 : espèce couvrant 75 à $100 \%$ de la surface du relevé, soit un recouvrement moyen de $87,5 \%$;

4 : espèce couvrant 50 à $75 \%$ de la surface du relevé, soit un recouvrement moyen de $62,5 \%$;

3 : espèce couvrant 25 à $50 \%$ de la surface du relevé, soit un recouvrement moyen de $37,5 \%$;

2 : espèce couvrant 5 à $25 \%$ de la surface du relevé, soit un recouvrement moyen de $15 \%$;

1 : espèce couvrant 1 à $5 \%$ de la surface du relevé, soit un recouvrement moyen de $3 \%$;

+ : espèce couvrant 0 à $1 \%$ de la surface du relevé, soit un recouvrement moyen de $0,5 \%$.

Le traitement des données phytosociologiques a été réalisé dans le tableur Excel du logiciel Microsoft Office 2007. Dans le cadre de cette étude, les types biologiques ont été aussi identifiés selon la classification écologique de Raunkiaer (1934). Par leur répartition, ils traduisent fidèlement les conditions écologiques d'une région. Leur étude permet d'avoir une idée de la végétation d'une région donnée (Mahamane, 2005). Ils désignent le comportement adaptatif des espèces végétales suivant la position des bourgeons et la taille des individus.

L'étude des spectres écologiques a été aussi réalisée et a permis l'appréciation des stratégies de vie (types biologiques) et l'étude de la distribution géographique 
des espèces (types phytogéographiques) (Oumarou, 2003 ; Mahamane, 2005). Ainsi, on distingue :

Le Spectre brut qui est le rapport en pourcentage entre l'effectif cumulé d'un type phytogéographique et l'effectif cumulé des espèces de tous les types phytogéographiques ;

le Spectre pondéré qui est le rapport en pourcentage entre le recouvrement moyen cumulé (Coefficient d'Abondance-Dominance) d'un type phytogéographique et le recouvrement cumulé des espèces de tous les types phytogéographiques.

Le spectre phytogéographique exprime le niveau de modification de la composition d'une communauté végétale ou d'un pâturage en ce sens que le regroupement des espèces selon leur chorologie est un élément essentiel de la compréhension du fonctionnement des écosystèmes (Soumana, 2012).

Les données dendrométriques collectées à travers l'inventaire ont permis de calculer les paramètres suivants :

Le taux de survie, qui correspond au rapport en pourcentage entre le nombre de plants ayant survécu au moins une année après la plantation et le nombre total de plants plantés ;

L'accroissement moyen annuel (en hauteur et en diamètre) des espèces plantées. On entend par

\section{RÉSULTATS}

Occupation du sol: L'interprétation des images Landsat de 2001 et de 2017a mis en évidence les changements intervenus au niveau des unités accroissement la quantité de matière fabriquée par unité de temps. Cette unité, employée en gestion forestière, est l'année qui correspond à l'échelle de temps où le phénomène est observé au mieux. On estime l'accroissement annuel en diamètre (ou en hauteur) d'un arbre en divisant la différence entre deux mesure successives du diamètre (ou de la hauteur) par l'intervalle de temps séparant ces mesures (Lompo, 1999).Dans le cadre de cette étude, la différence obtenue entre les mesures de hauteur et de diamètre effectuées sur les espèces plantées lors de l'inventaire et à la plantation, divisé par 2 ans (âge des arbres plantés) a été considérée comme l'accroissement annuel.

La densité des ligneux sur le site, qui exprime le nombre d'individus considérés dans l'inventaire par unité de surface ramené à l'hectare (Youssifi, 2014). C'est un indice biologique qui renseigne sur l'abondance des individus d'une espèce dans un site donné ;

Le recouvrement des ligneux qui correspond à la surface du sol qui serait recouverte par la projection des parties aériennes des individus de l'espèce (Youssifi, 2014). II renseigne sur l'évolution du peuplement sous les effets anthropiques (Karim, 2001).

d'occupation de sol sur toute la superficie du site d'étude (Figure 3). 


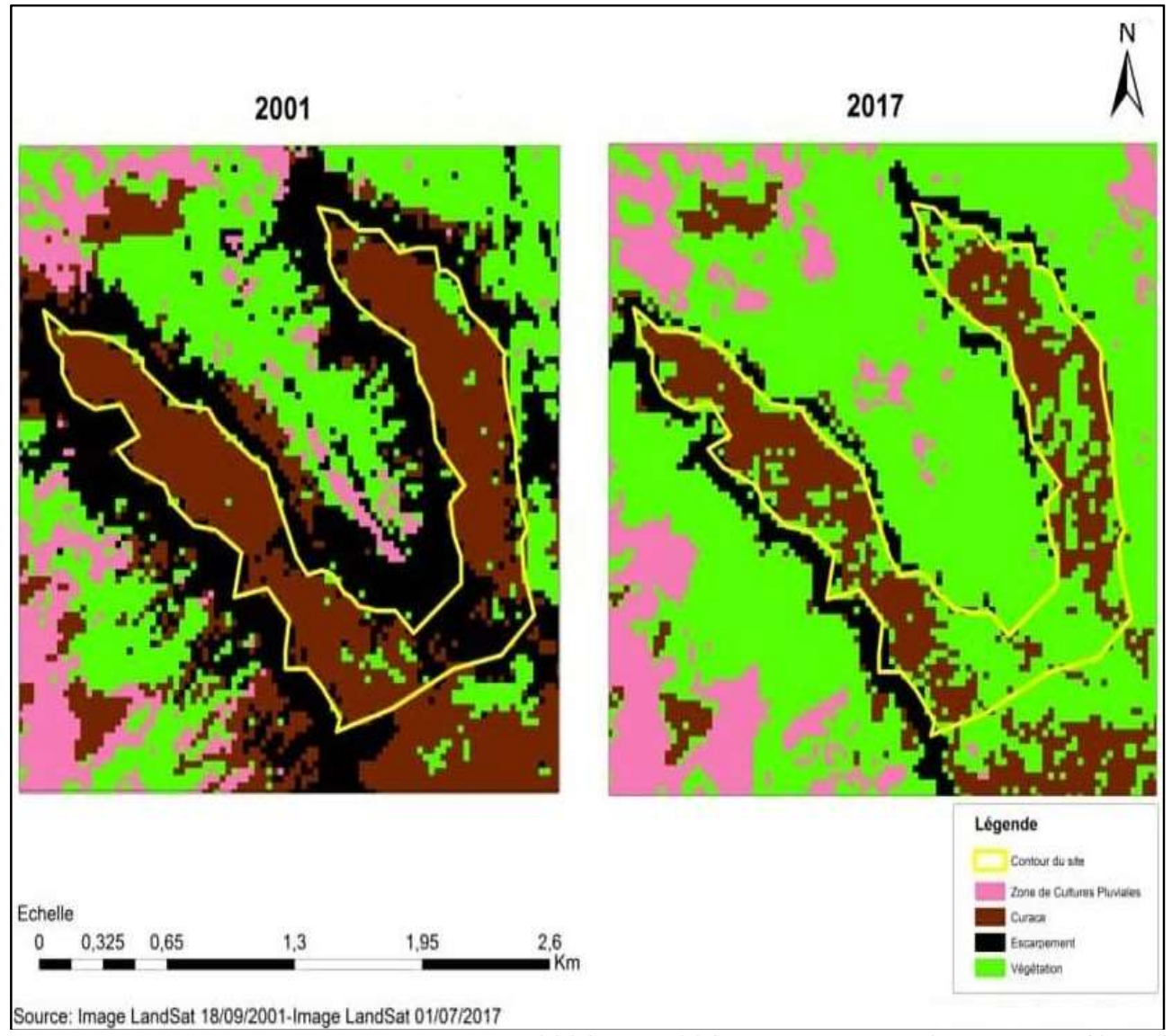

Figure 3 : Images d'occupation de sols de 2001 et de 2017 du plateau de Sini Bangou (Simiri)

II a été observé un recouvrement beaucoup plus important en 2017 attribuable à l'augmentation du couvert végétal de l'ordre de 17,24 ha, soit $11,50 \%$ de la superficie totale du plateau (Tableau 1). Cette augmentation pourrait s'expliquer par les effets induits par les actions de récupération des terres effectuées en 2015 sur la végétation.

Tableau1 : Evolution de l'occupation de sol du plateau de Sini Bangou (Simiri) entre 2001 et 2017

\begin{tabular}{l|c|c|c|c}
\hline \multirow{2}{*}{ Année } & \multicolumn{2}{|c|}{ Sol nu } & \multicolumn{2}{c}{ Couvert végétal } \\
\cline { 2 - 5 } & Superficie (ha) & Proportion (\%) & Superficie (ha) & Proportion (\%) \\
\hline $\mathbf{2 0 0 1}$ & 146,87 & 97,91 & 3,13 & 2,08 \\
$\mathbf{2 0 1 7}$ & 129,63 & 86,42 & 20,37 & 13,58 \\
Ecart 2001-2017 & & & 17,24 & 11,50 \\
\hline
\end{tabular}

Caractérisation du sol

Caractéristiques granulométriques du sol: Les résultats des analyses granulométriques des échantillons de sol (tableau 4) montrent une texture limono-sableuse dans le bloc1 au niveau des deux horizons $(0-20 \mathrm{~cm}$ et $20-40 \mathrm{~cm})$ en dehors de l'impluvium (Tableau 2). Dans le bloc2, la texture sablolimoneuse est prédominante dans les horizons de 20 à $40 \mathrm{~cm}$. 
Tableau 2 : Caractéristiques granulométriques des sols du plateau de Sini Bangou (Simiri)

\begin{tabular}{l|l|l|l|l|l|l|l}
\hline Blocs & $\begin{array}{l}\text { Zone de } \\
\text { prélèvement }\end{array}$ & $\begin{array}{l}\text { Profondeur } \\
(\mathbf{c m})\end{array}$ & Argile $(\mathbf{\%})$ & $\begin{array}{l}\text { Limon } \\
(\%)\end{array}$ & $\begin{array}{l}\text { Sable } \\
(\%)\end{array}$ & $\begin{array}{l}\text { Diamètre } \\
\text { moyen }(\boldsymbol{\mu m})\end{array}$ & Statut textural \\
\hline Bloc 1 & Tranchée & $0-20$ & 9,18 & 30,52 & 60,30 & 179 & Limono-sableux \\
& & $20-40$ & 10,90 & 30,86 & 58,24 & 174 & Limono-sableux \\
& Scarifiée & $0-20$ & 9,37 & 28,69 & 61,93 & 180 & Limono-sableux \\
& Impluvium & $0-20$ & 8,85 & 31,76 & 59,39 & 175 & Limono-sableux \\
& & $20-40$ & 8,79 & 24,10 & 67,10 & 199 & Limono-sableux \\
& Tranchée & $0-20$ & 11,14 & 22,52 & 66,34 & 194 & Limono-sableux \\
& & & & & & & \\
Bloc 2 & & & & & & & \\
& Scarifiée & $0-20$ & 7,62 & 17,91 & 75,47 & 232 & Limono-sableux \\
& & $20-40$ & 6,18 & 17,15 & 75,62 & 233 & Limono-sableux \\
& Impluvium & $0-20$ & 7,34 & 20,82 & 73,00 & 242 & Limono-sableux \\
& & $20-40$ & 6,80 & 26,37 & 66,29 & 204 & Limono-sableux \\
& & 20,22 & 69,98 & 227 & Limono-sableux \\
\hline
\end{tabular}

Dans les deux blocs, la proportion de sable reste la plus élevée.

Dépôt de sédiments: L'analyse des résultats de mesures de l'épaisseur des dépôts sédimentaires au niveau des tranchées, dans les deux blocs 1 et 2 considérés pour cette expérimentation, montre que celle-ci varie selon les banquettes (Tableau 3).En considérant les mesures dans les deux blocs, la moyenne d'épaisseur est de $9 \mathrm{~cm}$ dans le bloc 1 et 15 $\mathrm{cm}$ dans le bloc 2.

Tableau 3: Caractéristiques granulométriques des dépôts de sédiments

\begin{tabular}{|c|c|c|c|c|c|c|c|}
\hline $\begin{array}{l}\text { Zone de } \\
\text { prélèvement } \\
\text { (Tranchée) }\end{array}$ & $\begin{array}{l}\text { Epaisseur } \\
\text { des dépôts } \\
\text { (cm) }\end{array}$ & $\begin{array}{c}\text { argile } \\
(\%)\end{array}$ & $\begin{array}{c}\operatorname{limon}_{(\%)} \\
\text { (\%) }\end{array}$ & $\begin{array}{c}\text { sable } \\
(\%)\end{array}$ & $\begin{array}{c}\text { diamètre } \\
\text { moyen } \\
(\mu \mathrm{m})\end{array}$ & Statut textural & $\begin{array}{c}\text { Fraction > } \\
\text { à } 2000 \mu \mathrm{m} \\
(\%)\end{array}$ \\
\hline Bloc1 & $0-8$ & 11,18 & 29,77 & 59,06 & 163 & Limono-sableux & 18,32 \\
\hline Bloc1 & $0-15$ & 9,37 & 27,94 & 62,68 & 173 & Limono-sableux & 36,4 \\
\hline Bloc2 & $0-10$ & 10,24 & 20,99 & 68,77 & 193 & Limono-sableux & 41,25 \\
\hline
\end{tabular}

Proportion de la matière organique : Les résultats des analyses de la teneur du sol en carbone organique et en matière organique (Tableaux 4 et 5) montrent que, dans les horizons de 0 à $20 \mathrm{~cm}$, elle est relativement élevée dans toutes les parties de la banquette avec des valeurs maximale de plus de 1,11; 1,03 et $1,43 \%$ respectivement au niveau de la tranchée des deux blocs 1 et 2 et des sédiments.

Tableau 4: Teneur du sol en matière organique et en carbone organique des blocs 1 et 2

\begin{tabular}{c|c|c|c|c}
\hline Blocs & $\begin{array}{c}\text { Zone de } \\
\text { prélèvement }\end{array}$ & $\begin{array}{c}\text { Profondeur } \\
(\mathbf{c m})\end{array}$ & $\begin{array}{c}\text { Carbone organique } \\
(\%)\end{array}$ & Matière organique (\%) \\
\hline \multirow{5}{*}{ Bloc 1 } & Tranchée & $0-20$ & 0,64 & 1,11 \\
& & $20-40$ & 0,43 & 0,73 \\
& Scarifiée & $0-20$ & 0,37 & 0,64 \\
& & $20-40$ & 0,24 & 0,42 \\
& Impluvium & $0-20$ & 0,28 & 0,48 \\
& & 20 & 0,23 & 0,39 \\
Bloc 2 & Tranchée & $0-20$ & 0,60 & 1,03 \\
& & $20-40$ & 0,37 & 0,64 \\
& \multirow{3}{*}{ Scarifiée } & $0-20$ & 0,34 & 0,59 \\
& & $20-40$ & 0,27 & 0,47 \\
& \multirow{2}{*}{ Impluvium } & $0-20$ & 0,23 & 0,40 \\
\hline
\end{tabular}


Les résultats révèlent aussi que les proportions de la teneur en carbone organique et en matière organique varient en fonction de l'épaisseur du dépôt sédimentaire (tableau 5).

Tableau 5 : Teneur en matière organique et en carbone organique des dépôts sédimentaires des blocs

\begin{tabular}{l|c|c|c}
\hline Zone de prélèvement & $\begin{array}{c}\text { Epaisseur } \\
\text { du dépôt }(\mathbf{c m})\end{array}$ & Carbone organique (\%) & Matière organique (\%) \\
\hline Tranchée (Bloc1) & $0-8$ & 0,83 & 1,43 \\
Tranchée (Bloc1) & $0-15$ & 0,54 & 0,93 \\
Tranchée (Bloc2) & $0-10$ & 0,65 & 1,11 \\
Tranchée (Bloc3) & $0-15$ & 0,55 & 0,94 \\
\hline
\end{tabular}

Ainsi, elles sont plus importantes au niveau des horizons de surface de $0-20 \mathrm{~cm}$ et faibles en profondeur $(20-40 \mathrm{~cm})$. Au niveau des sédiments, on observe la même situation, mais avec des proportions de teneurs, en carbone organique et en matière organique, plus élevées.

Fonctionnement hydrique : Les résultats de l'analyse du fonctionnement hydrique dans les trois entités des banquettes (tranchée, partie scarifiée et impluvium) des blocs 1, 2 et 3 montrent que la vitesse d'infiltration est relativement lente pendant les dix premières minutes $(\leq$ $10 \mathrm{~cm} / \mathrm{mn}$ ) et l'infiltration ne dépasse guère les $10 \mathrm{~cm}$ de profondeur (Figures 4, 5 et 6 ) et, pour tous les cas étudiés. Pour les intervalles de temps de 5 minutes, l'infiltration est relativement constante avec en moyenne $1 \mathrm{~cm} / \mathrm{mn}$ de vitesse.

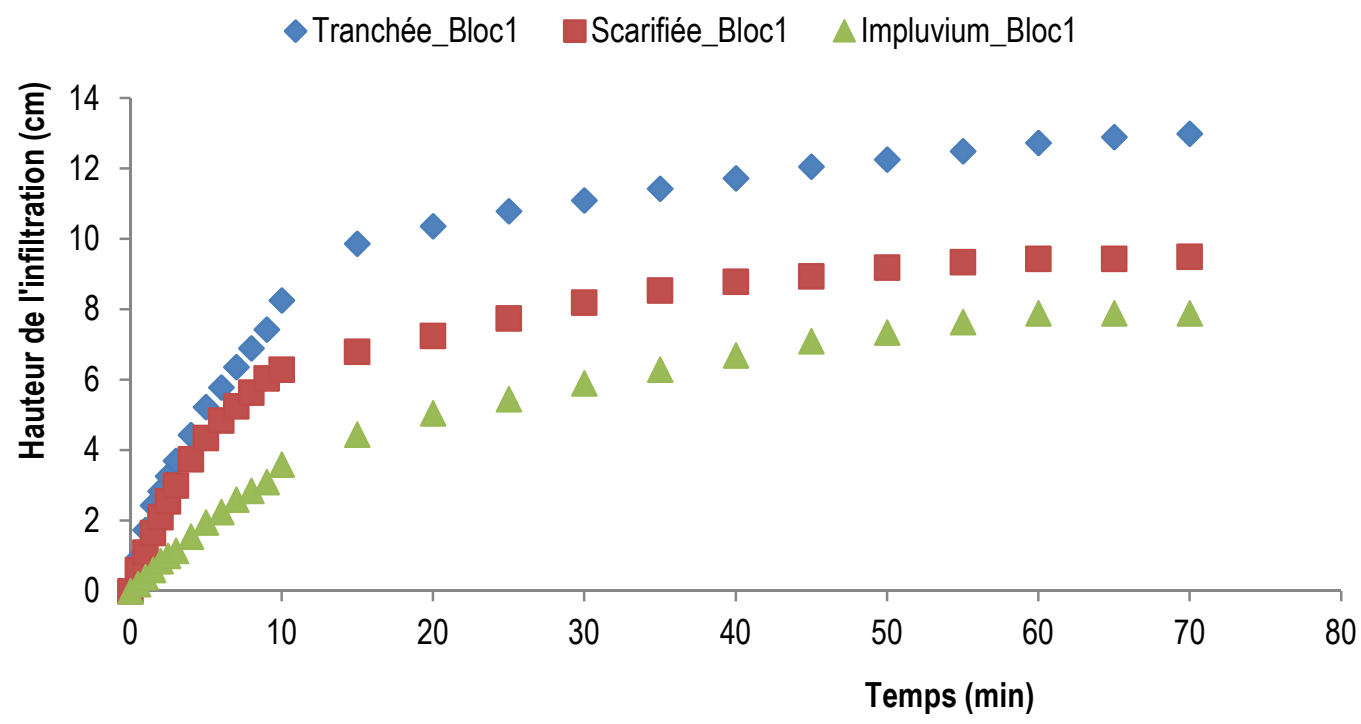

Figure4: Infiltration moyenne au niveau du bloc 1 


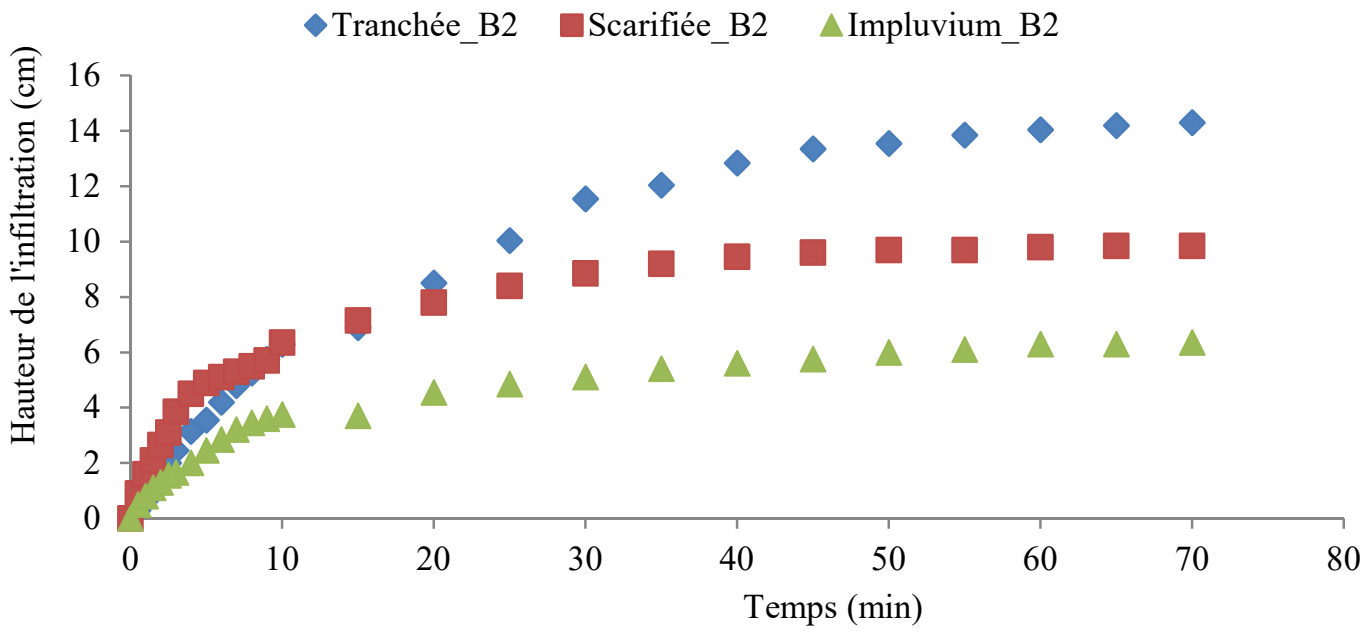

Figure5 : Infiltration moyenne au niveau du bloc 2

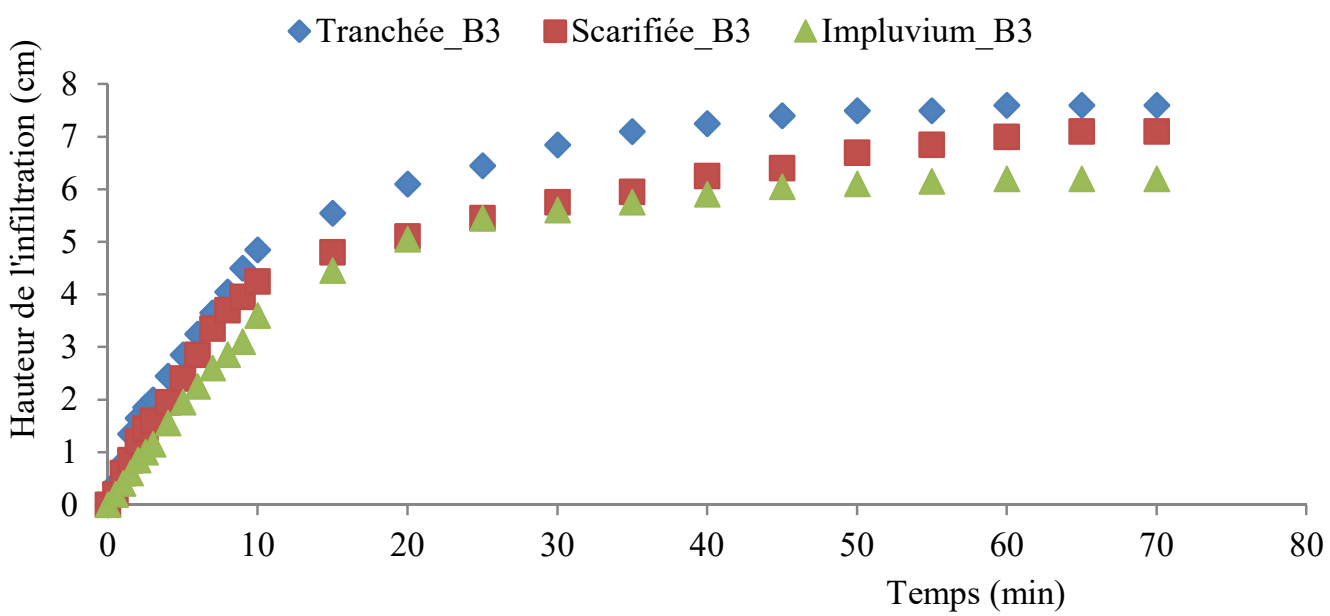

Figure6: Infiltration moyenne au niveau du bloc 3

Pendant les 70 minutes de la durée du test, l'infiltration a progressé au rythme d'une moyenne de $1 \mathrm{~cm} / \mathrm{mn}$. Cette progression en profondeur a cessé au bout des 30 minutes après le début du test et une infiltration latérale a pris le relais. Aussi, il est à noter quel infiltration est arithmétiquement plus élevée dans la tranchée que dans la partie scarifiée et est moindre dans l'impluvium au niveau des trois blocs.

Caractérisation de la végétation

Composition floristique: Les résultats de l'inventaire floristique montrent que le site d'étude compte 69 espèces végétales (Tableau 6). 
Tableau1: Liste floristique du site d'étude

\begin{tabular}{|c|c|c|c|c|}
\hline $\mathbf{N}^{\circ}$ & Espèces & Familles & Type biologique & $\begin{array}{l}\text { Chorologie } \\
\text { Type phytogéo } \\
\text { graphique }\end{array}$ \\
\hline 1 & Acacia holosericea A. Cunn. Ex G. don & Mimosaceae & $\mathrm{McPh}$ & GC SZ \\
\hline 2 & Acacia raddiana (Savi) Brenan & Mimosaceae & $\mathrm{McPh}$ & SZ-Sah.S \\
\hline 3 & Acacia senegal (L.) Willd & Mimosaceae & $\mathrm{McPh}$ & SZ \\
\hline 4 & Alysicarpus ovalifolius (Schum. et Thonn.) J. Leonard & Fabaceae & Th & GC SZ \\
\hline 5 & Andropogon gayanus Kunth Var gayanus & Poaceae & $\mathrm{He}$ & $\mathrm{SZ}$ \\
\hline 6 & Aristida funiculata Trin. Et Rupr. & Poaceae & Th & SZ \\
\hline 7 & Aristida mutabilis Trin. Et Rupr. & Poaceae & Th & SZ \\
\hline 8 & Borreria radiata DC. & Rubiaceae & Th & GC SZ \\
\hline 9 & Borreria stachydea (DC.) Hutch. Et Dalz & Rubiaceae & Th & GC SZ \\
\hline 10 & Boscia senegalensis (Pers.) Lam. Ex Poir. & Capparidaceae & $\mathrm{McPh}$ & SZ-Sah.S \\
\hline 11 & Brachiaria villosa (Lam.) A. Camus. & Poaceae & Th & GC SZ Sah S \\
\hline 12 & Brachiaria ramosa (L.) Stapf & Poaceae & Th & SZ \\
\hline 13 & Calotropis procera (Ait.) R. Br. & Asclepiadaceae & $\mathrm{McPh}$ & GC SZ Sah S \\
\hline 14 & Cassia mimosoïdes L. & Caesalpiniaceae & Th & GC-SZ-Sah.S \\
\hline 15 & Cassia nigricans Vahl & Caesalpiniaceae & Th & SZ-Sah.S \\
\hline 16 & Cenchrus biflorus Roxb & Poaceae & Th & GC-SZ-Sah.S \\
\hline 17 & Cenchrus prieuri (Kunth.) Maire & Poaceae & Th & SZ-Sah.S \\
\hline 18 & Ceratotheca sesamoides Endl. & Pedaliaceae & Th & SZ \\
\hline 19 & Chloris prieurri kunth. & Poaceae & Th & SZ-Sah.S \\
\hline 20 & Chrozophora brocchiana Vis. & Euphorbiaceae & $\mathrm{NnPh}$ & SZ-Sah.S \\
\hline 21 & Citrillus lanatus (Thunb.)Matsumara et Nakai & Cucurbitaceae & Th & SZ-Sah.S \\
\hline 22 & Combretum micranthum G. Don. & Combretaceae & $\mathrm{McPh}$ & SZ \\
\hline 23 & Crotalaria atrorubens Hochst.Ex Benth & Fabaceae & Th & $S Z$ \\
\hline 24 & Crotalaria pallida Ait et L. & Fabaceae & Th & GC-SZ \\
\hline 25 & Cténium elegans Kunth. & Poaceae & Th & $\mathrm{SZ}$ \\
\hline 26 & Cymbopogon giganteus Chiov. & Poaceae & $\mathrm{He}$ & GC-SZ \\
\hline 27 & Cymbopogon schoenanthus (L.) Spreng & Poaceae & $\mathrm{He}$ & SZ Sah S \\
\hline 28 & Cyperus conglomeratus Rottb. & Cyperaceae & $\mathrm{He}$ & SZ-Sah.S \\
\hline 29 & Dactyloctenium aegyptium (L.) Willd. & Cyperaceae & Th & GC-SZ-Sah.S \\
\hline 30 & Dicoma tomentosa Cass. & Asteraceae & Th & SZ \\
\hline 31 & Digitaria gayana (Kunth) A.Chev. & Poaceae & Th & GC SZ \\
\hline 32 & Digitaria horizontalis Willd. & Poaceae & Th & GC SZ \\
\hline 33 & Eragrostis tremula Hochst. Ex Steud. & Poaceae & Th & GC-SZ-Sah.S \\
\hline 34 & Eucalyptus camaldulensis Dehnh. & Myrtaceae & $\mathrm{MsPh}$ & $\mathrm{i}$ \\
\hline 35 & Evolvulus alsinoïdes (L.) L. & Convolvulaceae & Th & GC-SZ-Sah.S \\
\hline 36 & Fimbristylis exilis Humb., Bonpl. et Kunth & Cyperaceae & Th & GC SZ \\
\hline 37 & Guiera senegalensis J.F. Gmel. & Combretaceae & $\mathrm{McPh}$ & $\mathrm{SZ}$ \\
\hline 38 & Hibiscus asper Hook. f. & Malvaceae & Th & GC SZ \\
\hline 39 & Indigofera hirsuta L. var. hita & Fabaceae & Th & SZ-Sah.S \\
\hline 40 & Indigofera senegalensis Lam. & Fabaceae & Th & SZ-Sah.S \\
\hline 41 & Ipomoea involucrataP. Beauv. & Convolvulaceae & Th & GC SZ \\
\hline 42 & Ipomoea vagans Bak & Convolvulaceae & Th & SZ \\
\hline 43 & Jacquemontia tamnifolia (L.) Griseb. & Convolvulaceae & Th & GC SZ \\
\hline 44 & Leptadenia hastata (Pers.) Decne & Asclepiadaceae & $\mathrm{McPh}$ & SZ-Sah.S \\
\hline 45 & Merremia pinnata (Hochst. ex Choisy) Hallier f & Convolvulaceae & Th & GC SZ \\
\hline 46 & Merremia tridentata (L.) Hallier & Convolvulaceae & Th & GC SZ \\
\hline 47 & Mitracarpus scaber Zucc. & Rubiaceae & Th & $\mathrm{SZ}$ \\
\hline
\end{tabular}




\begin{tabular}{l|l|l|l|l}
\hline 48 & Mollugo nudicaulis Lam. & Mulluginaceae & Th & GC SZ \\
49 & Monechma ciliatum (Jacq.) Milne. Redhead & Acanthaceae & Th & GC SZ \\
50 & Pancratium trianthum (Herbert) & Amaryllidaceae & Gb & GC-SZ \\
51 & Pandiaka involucrata (Moq.) B. D. Jacqueson. & Amaranthaceae & Th & SZ \\
52 & Panicum laetum Kunth. & Poaceae & Th & GC SZ \\
53 & panicum subalbidum Kunth. & Poaceae & Th & GC SZ \\
54 & Panicum anabaptistum Steud. & Poaceae & He & SZ \\
55 & Pennisetum pedicellatum Trin. & Poaceae & Th & GC-SZ-Sah.S \\
56 & Piliostigma reticulatum (DC.) Hochst. & Caesalpiniaceae & McPh & SZ-Sah.S \\
57 & Polycarpaea linearifolia (DC.) DC & Caryophyllaceae & Th & SZ-Sah.S \\
58 & Prosopis juliflora (Sw) DC & Mimosaceae & MsPh & i \\
59 & Schizachyrium exile (Hochst) Pilger. & Poaceae & Th & GC-SZ-Sah.S \\
60 & Schoenefeldia gracilis Kunth. & Poaceae & Th & GC-SZ-Sah.S \\
61 & Sesbania leptocarpa DC. & Fabaceae & Th & GC-SZ-Sah.S \\
62 & Sida cordifolia L. & Malvaceae & Ch & GC SZ \\
63 & Sida ovata Forsk. & Malvaceae & Ch & SZ \\
64 & Tephrosia bracteolata Gill. Et Perr & Fabaceae & Th & SZ \\
65 & Tephrosia linearis (Will.) Pers. & Fabaceae & Th & SZ \\
66 & Tephrosia purpurea (L.) Pers. & Fabaceae & Ch & SZ-Sah.S \\
67 & Waltheria indica L. & Sterculiaceae & NnPh & GC SZ \\
68 & Ziziphus mauritiana Lam. & Rhamnaceae & McPh & SZ-Sah.S \\
69 & Zornia glochidiata Reichb. ex DC & Fabaceae & Th & GC SZ \\
\hline
\end{tabular}

Les espèces végétales recensées sont réparties entre 21 familles et 52 genres (Tableau 7). Les familles les plus représentées, par ordre décroissant, regroupent les Poaceae (22 espèces, soit $31,88 \%$ et 14 genres, soit $26,92 \%$ ), les Fabaceae-faboideae (12 espèces, soit $17,39 \%$ et 9 genres, soit $17,31 \%)$, les Convolvulaceae ( 6 espèces, soit $8,70 \%$ et 4 genres, soit $7,69 \%$ ), les Fabaceae-mimosoideae (4 espèces soit $5,88 \%$ et 2 genres, soit 3,85\%), les Mavalceae et les Rubiaceae ( 3 espèces, soit $4,35 \%$ et 2 genres, soit $3,85 \%$ chacune) et les Asclepiadaceae, les Fabaceaecaesalpinioideae, les Combretaceae et les Cyperaceae avec chacune 2 espèces, soit $2.90 \%$ et 2 genres, soit $3,85 \%$. Les autres familles sont représentées par 1 espèce et 1 genre.

Tableau 7: Nombre de genre et d'espèces de la végétation du site

\begin{tabular}{l|l|l|l|l}
\hline Famille & Nombre de Genres & \% de genre & Nombre d'espèces & \% d'espèces \\
\hline Acanthaceae & 1 & 1,92 & 1 & 1,45 \\
Amaranthaceae & 1 & 1,92 & 1 & 1,45 \\
Asclepiadaceae & 2 & 3,85 & 2 & 2,90 \\
Asteraceae & 1 & 1,92 & 1 & 1,45 \\
Fabaceae- caesalpinioideae & 2 & 3,85 & 2 & 2,90 \\
Capparaceae & 1 & 1,92 & 1 & 1,45 \\
Caryophyllaceae & 1 & 1,92 & 1 & 1,45 \\
Combretaceae & 2 & 3,85 & 2 & 2,90 \\
Convolvulaceae & 4 & 7,69 & 6 & 8,70 \\
Cucurbitaceae & 1 & 1,92 & 1 & 1,45 \\
Cyperaceae & 2 & 3,85 & 1 & 1,90 \\
Euphorbiaceae & 1 & 1,92 & 12 & 17,39 \\
Fabaceae-faboideae & 9 & 17,31 & 3 & 4,35 \\
Malvaceae & 2 & 3,85 & 4 & 5,80 \\
Fabaceae-mimosoideae & 2 & 3,85 & 1 & 1,45 \\
Molluginaceae & 1 & 1,92 & 1 & 1,45 \\
Myrtaceae & 1 & 1,92 & & \\
\hline
\end{tabular}




\begin{tabular}{l|l|l|l|l}
\hline Poaceae & 14 & 26,92 & 22 & 31,88 \\
Rhamnaceae & 1 & 1,92 & 1 & 1,45 \\
Rubiaceae & 2 & 3,85 & 3 & 4,35 \\
Sterculiaceae & 1 & 1,92 & 1 & 1,45 \\
Total & $\mathbf{5 2}$ & $\mathbf{1 0 0}$ & $\mathbf{6 9}$ & $\mathbf{1 0 0}$ \\
\hline
\end{tabular}

II est à noter que parmi les 69 espèces recensées, 5 espèces sont issues de plantation et de l'ensemencement. II s'agit des espèces Eucalyptus camaldulensis (Myrtaceae) et Acacia senegal (Mimosaceae), Pennisetum pedicellatum, Cymbopogon schoenanthus et Cenchrus biflorus (Poaceae). Toutes les autres espèces sont issues de la régénération naturelle à la faveur des travaux de récupération des terres réalisés. La densité globale des ligneux du site est de 84 pieds/ha. Elle est respectivement de 33 pieds/ha pour Guiera senegalensis, 17 pieds/ha pour
Boscia senegalensis, 14 pieds/ha pour Eucalyptus camaldulensis, 12 pieds/ha pour Acacia senegal, 5 pieds/ha pour Combretum micranthum et 2 pieds/ha pour Piliostigma reticulatum. Dans le recouvrement, il a été considéré les arbres issus de la plantation et de la régénération naturelle avec l'objectif est d'évaluer la contribution de chaque groupe d'arbres dans le recouvrement du site. Le tableau 8 donne les recouvrements moyens des différents groupes d'arbres en fonction de leur origine.

Tableau 8 : Recouvrement des ligneux sur le site

\begin{tabular}{l|c|c|c|c}
\hline & $\begin{array}{c}\text { Arbres } \\
\text { plantés }\end{array}$ & $\begin{array}{c}\text { Arbres } \\
\text { naturels }\end{array}$ & $\begin{array}{c}\text { Arbres } \\
\text { d'origine mixte }\end{array}$ & $\begin{array}{c}\text { Recouvrement } \\
\text { total }\end{array}$ \\
\hline Recouvrement moyen (\%) & $1,88 \pm 5,47$ & $3,26 \pm 7,39$ & $2,17 \pm 5,51$ & 7,31 \\
\hline
\end{tabular}

L'analyse des données issues de l'inventaire dendrométrique, montre un recouvrement moyen des ligneux de $7,31 \%$ dont $1,88 \%$ pour les plantations, $3,26 \%$ pour la régénération naturelle et $2,17 \%$ pour les peuplements regroupant les arbres issus de la plantation et de la régénération naturelle.
Types biologiques: L'analyse des types biologiques (Figure 7), montre que les Thérophytes (Th) constituent essentiel de la forme biologique de cette flore avec $69,01 \%$ de spectre pondéré et $66,67 \%$ de spectre brut.

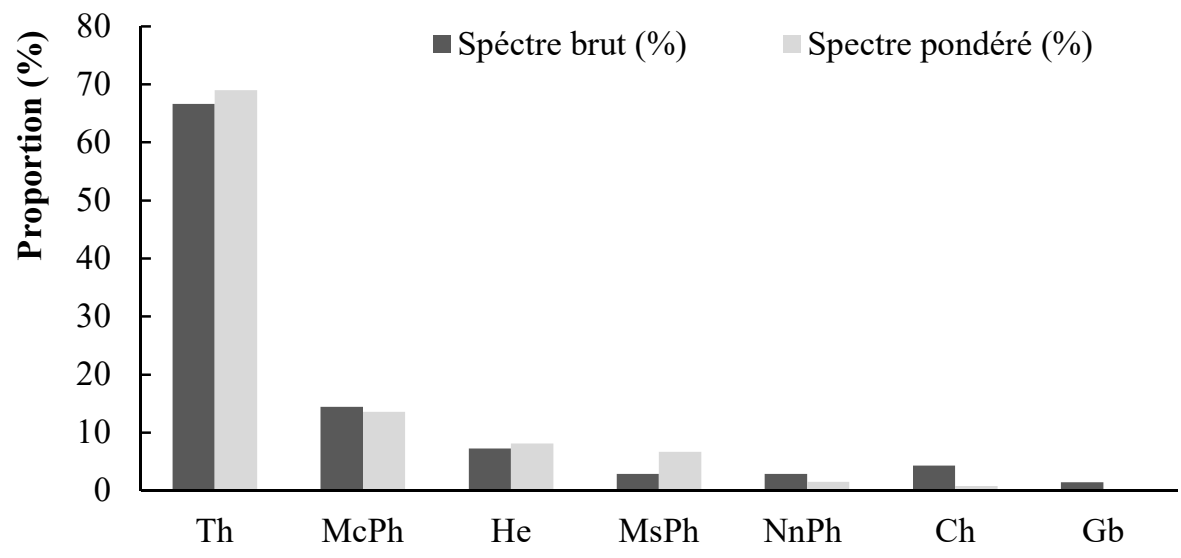

Types biologiques

Figure7: Types biologiques des espèces recensées

Légende: Th: Thérophytes, McPh: Microphanérophites, He: Hemicryptophytes, MsPh : Mésophanérophytes, NnPh: Nanophanérophytes, $\mathrm{Ch}$ : Chaméphytes, Gb : Géophytes bulbeux 
Elles sont suivies par les Microphanérophites (McPh), les Hemicryptophytes $(\mathrm{He})$, les Mésophanérophytes (MsPh), les Nanophanérophytes ( $\mathrm{NnPh})$, les Chaméphytes (Ch) et les Géophytes bulbeux (Gb) avec respectivement $13,58 \% ; 8,11 \% ; 6,70 \% ; 1,53 \% ; 0,81 \%$, $0,26 \%$ de spectre pondéré. Les types biologiques les moins représentés sont Géophytes bulbeux (Gb) avec $0,26 \%$ de spectre pondéré et $1,45 \%$ de spectre brut.

Types phytogéographiques: L'analyse phytogéographique (Figure 8) montre que, à l'échelle africaine, la flore du site est dominée par les espèces Soudano-Zambéziennes avec $33,21 \%$ de spectre pondéré et $21,18 \%$ de spectre brut.

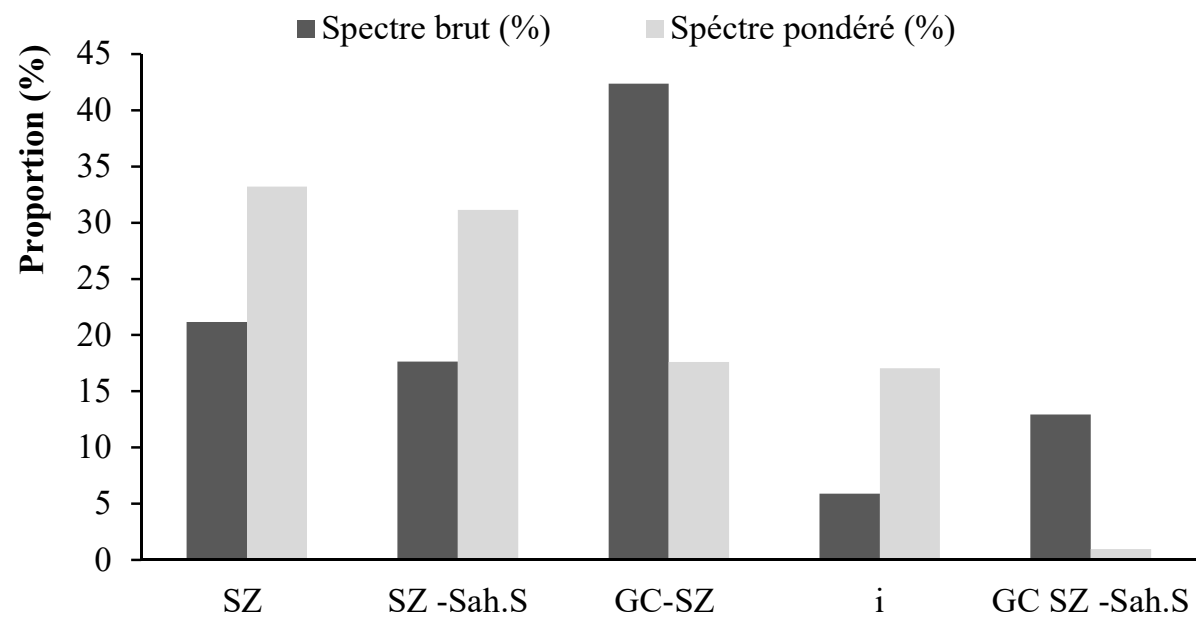

Types phytogéographiques

Figure8: Analyse globale des types phytogéographiques

Légende: GC-SZ: Guinéo-Congolaise-Soudano-Zambéziennes; SZ: Soudano-Zambéziennes; GC-SZ-S: GuinéoCongolaise-Soudano-Zambéziennes-Sindiennes ; GC-SZ-Sah-S: Guinéo-Congolaise-Soudano-Zambéziennes-SahariennSindiennes ; Pal : Paléotropicale ; SZ-Sah-S : Soudano-Zambéziennes-Saharo-Sindiennes.

Elles sont suivies des espèces SoudanoZambéziennes-Saharo-Sindiennes $(31,15 \%$ de spectre pondéré et $17,65 \%$ de spectre brut), des GuinéoCongolaise-Soudano-Zambéziennes $\quad(17,61 \%$ de spectre pondéré et $42,35 \%$ de spectre brut), des espèces introduites $(17,06 \%$ de spectre pondéré et $5,88 \%$ de spectre brut) et des espèces GuinéoCongolaise-Soudano-Zambéziennes-SahariennesSindiennes avec $0,98 \%$ de spectre pondéré et $12,94 \%$ de spectre brut qui, sont le type le moins représenté.
Taux de survie et de croissance de la plantation : Le taux de survie, deux années après la plantation, est de 64,44et 66,88\% respectivement pour les espèces Eucalyptus camaldulensis et Acacia senegal, soit un taux de survie global de 65,66\%. L'analyse des résultats de la croissance des espèces plantées donne une croissance moyenne annuelle en hauteur de 130,5 $\pm 0,22$ et $33 \pm 0,47 \mathrm{~cm}$ et en diamètre de 1,51 $\pm 0,36$ et $0,74 \pm 0,18 \mathrm{~cm}$ respectivement pour $E$. camaldulensis et A. senegal (Tableau 9).

Tableau9 : Evolution dendrométrique moyenne des espèces issues de la plantation

\begin{tabular}{l|c|c}
\hline Espèces & $\begin{array}{c}\text { Croissance moyenne annuelle } \\
\text { en hauteur }(\mathrm{cm})\end{array}$ & $\begin{array}{c}\text { Croissance moyenne annuelle en } \\
\text { diamètre }(\mathbf{c m})\end{array}$ \\
\hline Eucalyptus camaldulensis & $130,5 \pm 0,22$ & $1,51 \pm 0,36$ \\
Acacia senegal & $33,00 \pm 0,47$ & $0,74 \pm 0,18$ \\
\hline
\end{tabular}




\section{DISCUSSION}

Les résultats de l'analyse diachronique des images satellitaires sur l'occupation de sol ont mis en évidence, 16 ans après l'aménagement, une augmentation de couvert végétal de $11,5 \%$ de la superficie totale du site du plateau de Sini Bangou. En effet, les changements du couvert végétal, quantitatif ou qualitatif, sont les premiers éléments utilisés pour qualifier l'évolution d'un milieu en termes de dégradation, car ces éléments sont les plus facilement mesurables sur le terrain, à distance, par télédétection (Taîbi, 2015). Boubacar et al. (2013) ont fait aussi état d'un recouvrement moyen de $9,11 \%$ dans les systèmes sylvopastoraux des plateaux dégradés dans la même zone. Ces changements observés sont aussi illustrés par l'importance de la diversité floristique issue de la régénération naturelle dominée par les familles des Poaceae, des Fabaceae et des Convolvulaceae qui se sont développées à la faveur de l'aménagement réalisé. La dominance des Poaceae a été rapportée par plusieurs études menées au Sahel notamment celles de Mahamane (2005) dans le Parc régional du W du Niger, Morou (2010) dans région de Kouré, Kaou et al. (2017) dans celle de Mainé Soroa et Melom et al. (2015) dans la zone de Massenya au Tchad. Ce qui confirme que les Poaceae sont caractéristiques de la zone sahélienne. Les résultats de l'étude intègrent ceux de Manirou (2012), plus globaux, qui a travaillé sur l'évaluation de statut de conservation des espèces végétales à l'échelle de la commune rurale de Simiri. Ceci illustre que l'aménagement a induit une réhabilitation du couvert végétal dont la composition floristique tend vers celle de la végétation naturelle. Cependant, la prédominance des Thérophytes s'explique par le caractère saisonnier de leur apparition, leur adaptation au milieu aride et semi-aride, ainsi que le niveau de dégradation avancée du milieu (Saadou, 1990). Selon Mahamane et al. (2009), des telles caractéristiques structurales du couvert végétal traduisent un milieu assez ouvert et sec, marqué par une dégradation très marquée par les pratiques sylvopastorales. Ces résultats sont confortés par l'étude de Manirou (2012) qui a trouvé des résultats similaires à l'échelle globale de la commune rurale de Simiri et d'autres études dans les régions du Sahel notamment à Kouré (Morou, 2010), à Gouré (Abdou, 2016) et à Mainé Soroa (Kaou et al., 2017) au Niger et à Assaba en Mauritanie (Abdellahi et al., 2011). La répartition phytogéographique des espèces montre une plus grande distribution des espèces soudanozambéziennes suivies des espèces soudano- zambéziennes-saharo-sindiennes dans le spectre pondéré alors que les espèces guinéo-congolaisessoudano-zambéziennes montrent une plus large distribution dans le spectre brut. En effet, les deux groupes d'espèces, soudano-zambéziennes et guinéocongolaises-soudano-zambéziennes, même s'ils révèlent une modification par rapport aux observations de Saadou (1990), sont les plus largement distribuées en zone sahélienne (Saadou, 1990 ; Manirou, 2012) et confirment l'adaptation des espèces au caractère aride du plateau. En effet, la présence d'espèces plurirégionales témoigne d'une végétation perturbée. La forte proportion des espèces à large distribution est un indice de perturbation et indique que la flore perd de sa spécificité (Sinsin, 1993 ; Sinsin, 2001).La dégradation du milieu consécutif aux pressions anthropiques et climatiques est probablement la cause de la baisse de proportion de ces espèces. II est évident que cette évolution de la couverture végétale du site annonce une dynamique de changement positif de la flore sur le site qui est assimilé à un impact positif de l'aménagement réalisé. Les taux de survie et de croissance appréciables des arbres plantés ( $E$. camaldulensis et $A$. senegal) ont mis en évidence le rôle joué par les banquettes dans l'accumulation des sédiments, l'infiltration et le stockage des eaux. En effet, la survie et la croissance appréciables des plantations, malgré un sol peu favorable à un développement des racines en profondeur, sont dues au stockage saisonnier de l'eau dans les banquettes. Selon Noma (2017), la prospection racinaire varie entre 25 à $35 \mathrm{~cm}$ de profondeur. La densité, la grosseur, les ramifications sont très réduites et la racine principale présente des courbures justifiant les difficultés de pénétration. Selon Anonyme (1963), en conditions favorables, la croissance de l'E. camaldulensis peut être rapide, dépassant 3 mètres par an et atteignant 30 à $35 \mathrm{~m}$ en 10 ou 15 ans. Sur des stations pauvres, en terrain sec, la taille au même âge ne dépasserait probablement pas 15 à $18 \mathrm{~m}$. Les tests d'infiltration réalisés ont permis de mettre en évidence une infiltration plus ou moins rapide dans les horizons du sol au niveau des 10 premiers centimètres. Ceci est justifié par les résultats de la granulométrie des mêmes horizons du sol avec une dominance de sable. Au-delà de cette profondeur, malgré le faible niveau d'infiltration mesuré, l'impact sur le ruissellement et l'érosion est bien perceptible à travers la croissance des plants et les dépôts sédimentaires dans la tranchée. En effet, au niveau de cette dernière, l'eau s'infiltre plus facilement 
à cause de la couche de sable accumulée mais aussi de la densité d'arbres relativement importante. Lili et al. (2008) ont montré que la présence de la végétation est corrélée positivement avec l'infiltration. Cependant, la présence de la dalle à $50 \mathrm{~cm}$ de profondeur et souvent à 30 et $40 \mathrm{~cm}$ sur les plateaux latéritiques, limiterait considérablement l'infiltration (Karim, 2001). Ambouta et Dan Lamso (1996) ont montré que les sols de plateau sont pauvres en matière organique, en calcium, en phosphore assimilable, en capacité d'échange cationique (CEC). Cependant, le développement

\section{CONCLUSION}

L'objectif de l'étude était d'évaluer l'influence de banquettes anti-érosives dans l'amélioration de la couverture végétale et de la qualité du sol. La réalisation du dispositif des banquettes sur le plateau dégradé de Sini Bangou a enclenché des modifications agro-écologiques importantes au niveau du milieu. Ainsi, les banquettes ont permis une augmentation significative de la superficie couverte par la végétation, qui s'est traduite par une amélioration qualitative et quantitative de la richesse floristique et une régénération naturelle de plusieurs espèces ligneuses et herbacées. L'état structural et textural du sol s'est

\section{REMERCIEMENTS}

Les auteurs tiennent à remercier vivement le 'Projet Gestion des Risques et Catastrophes et Développement Urbain' (PGRC-DU) pour les moyens

\section{REFERENCES BIBLIOGRAPHIQUES}

Abdellahi OMV, Ahmed OIB, Ahmedou OS, Mathieu G.Jean N.L, 2011. Contribution à l'étude de la flore de Mauritanie : Évaluation de la biodiversité floristique de l'Assaba (Açaba) Mauritanie. Bulletin de l'Institut Scientifique, Rabat, section Sciences de la Vie 33 (2) : 5364.

Abdou MHK, 2016. Caractérisation de la végétation de la zone d'intervention du PLECO à Gouré. Mémoire de Master en Biodiversité et Gestion de l'Environnement Soudanien et SahéloSaharien, Faculté des Sciences et Techniques, Université Dan Dicko Dankoulodo de Maradi, 79p.

Ambouta KJM et Dan Lamso N, 1996. Rapport d'étude des sols des terroirs de Nazey et Togom (Ouallam). Faculté d'Agronomie, Université Abdou Moumouni de Niamey, $30 \mathrm{p}$. végétal au niveau de tranchée pourrait aussi être facilité par l'amélioration de la fertilité liée à l'évolution sensible, quoique faible, de la teneur en matière organique du sol associé aux proportions non négligeables de limon et de l'argile. Ce qui pourrait s'expliquer par une modification des propriétés physiques du sol à travers l'accumulation des sédiments, la décomposition de la biomasse, et enfin des conditions optimales pour une activité efficace de la microfaune (Blamah, 2004).

retrouvé aussi amélioré grâce au stockage de l'eau et le dépôt des sédiments dans les banquettes à la faveur d'une infiltration plus importante. Ce qui a permis l'installation de la végétation plus dense des espèces naturelles et favorisé la survie et la croissance appréciables des plantations réalisées. Les résultats de l'étude montrent que les travaux de récupération de terres à base des banquettes sont efficaces et pourraient même être un moyen efficace de réduction des risques climatiques à travers le développement des services éco-systémiques fournis par l'aménagement.

qu'il a acceptés de mettre dans la conduite de ce travail.

Anonyme, 1963.Eucalyptus camaldulensis Delmh et Eucalyptus tereticornis J.E.SM, TH, caractères sylvicoles et plantations, Revue Bois et Forêts des Tropiques $n^{\circ} 87,7 p$.

Banque Mondiale, 2013. Evaluation de projet de gestion des risques et catastrophes et d'aménagement urbain. Association Internationale de Développement, Rapport $n^{\circ}$ PAD817, version provisoire, $72 \mathrm{p}$.

Blamah J, 2004. Impact des Aménagements antiérosifs sur les Productions fourragères et le Recyclage des Nutriments au Sahel. Mémoire d'Ingénieur du Développement Rural en Elevage, Université Polytechnique de BoboDioulasso, $127 \mathrm{p}$.

Boubacar MM, Inoussa MM, Ambouta J.M.K, Mahamane A, Jorgen A, Harissou Y, Rabiou $\mathrm{H}$, 2013. Caractérisation de la végétation ligneuse et des organisations pelliculaires de 
surface des agroécosystèmes à différents stades de dégradation de la Commune rurale de Simiri (Niger).Int. J. Biol. Chem. Sci. 7(5) : 1963-1975

Braun-Blanquet J, 1932. Plant sociology. The study of plant communities. Ed. McGray Hill, New York, London, 439p.

CDR, 2016. Plan de Développement Communal 20172021 de la Commune Rurale de Simiri. Contribution au Développement Rural, rapport final, $113 p$.

Emily S, 2010. Les défis de la mise en œuvre de la Convention des Nations Unies sur la lutte contre la désertification. Essai présenté au département de biologie pour le grade de maître en écologie internationale, Faculté des Sciences, Département de Biologie Université de Sherbrooke, Québec, Canada, 60 p.

FAO, 1992. Guide pour l'interprétation des analyses de sol; Document de travail 13, AG : DP/SEN/87/Q07 ; $86 \mathrm{p}$.

FAO, 2010.Evaluation des ressources forestières mondiales. Rapport principal, Rome, Italie, $377 p$.

Hamidou SM, 2015. Impacts des actions de gestion durable des terres sur le milieu biophysique et le développement socio-économiques des populations rurales : cas de la grappe de Dembou Béri dans la zone d'intervention du PLCE/BN. Mémoire d'Inspecteur des Eaux et Forêts, Ecole Nationale des Eaux et Forêts de Bobo Dioulasso, Burkina Faso, $75 \mathrm{p}$.

INS, 2009.Populations des communes du Niger 20102012. Rapport final, Institut National de la Statistique du Niger, Ministère de l'Economie et des Finances, République du Niger

INS, 2012.Recensement Général de la Population et de l'Habitat du Niger 2011. Rapport final, Institut Nationale de la Statistique, République du Niger

Kaou KAK, Laminou MO, Dan Guimbo I, Karim S, Habou R, Paul R., 2017. Diversité floristique et structure de la végétation dans la zone dunaire du sud-est du Niger : Cas de Mainé soroa. J. Appl. Biosci. 120: 12053-12066

Karim S, 2001. Contribution à l'étude de la régénération par multiplication végétative naturelle de deux combretaceae dans l'ouest du Niger (Combretum micranthum G. Don et Guiera senegalensis J. F.Gmel : conséquences sur la gestion sylvopastorale. Mémoire de DEA en
Biologie et Ecologie Végétales, Université de Ouagadougou, $47 \mathrm{p}$.

Lili M, Bralts VF, Yinghua P, Han L, Tingwu L, 2008. Methods for measuring soil infiltration: State of the art. Int J Agric et Biol Eng 1(1) : 22-30

Lompo D, 1999. Etude de la croissance de quelques espèces ligneuses en plantation dans la forêt classée de Gonse Acacia albida Del., Azadirachta indica A. JUSS., Eucalyptus camaldulensis Delmh., Parkia biglobosa (Jacq.) Benth. et Ziziphus mauritiana Lam. Mémoire d'Ingénieur de Développement Rural, Option Eaux et Forêts, Université Polytechnique de Bobo-Dioulasso, $44 \mathrm{p}$.

Mahamane A, 2005. Etude floristique, phytosociologie et phytogéographique de la végétation du Parc Régional du W du Niger. Thèse de Doctorat en Sciences Agronomiques et Ingénierie Biologique, Université Libre de Bruxelles, Belgique, $516 \mathrm{p}$.

Mahamane A, Saadou M, Karim S, Yacoubou B, Morou B, Wata I, Diouf A, Mamane Maarouhi I, Issaka A, Tanimoune A, Soumana I, Jauffret S, 2009. Variabilité climatique au Niger : Impacts potentiels sur la distribution de la végétation. Actes du 14ème Colloque international en évaluation environnementale sur 'Changements climatiques et évaluation environnementale enjeux et outils pour l'évaluation des impacts et l'élaboration de plans d'adaptation', Niamey du 26 au 29 mai 2009.

Manirou O, 2012. Evaluation de statut de conservation des espèces végétales dans la commune rurale de Simiri (Niger). Mémoire de DEA en Biologie Appliquée, Option : "Protection et Restauration des Ecosystèmes Soudaniens et Sahelo-Sahariens », Faculté des Sciences et Techniques, Université Abdou Moumouni de Niamey, $56 \mathrm{p}$.

ME/SU/DD, 2014. Cadre stratégique de la gestion durable des terres au Niger et son plan d'investissement 2015-2029. Rapport final, Ministère de l'Environnement, de la Salubrité Urbaine et de du Développement Durable, République du Niger, $74 \mathrm{p}$.

Melom S, Mbayngone E, Bechir AB, Ratnan N, Mapongmetsem PM, 2015. Caractéristiques floristique et écologique des formations végétales de Massenya au Tchad (Afrique 
centrale). Journal of Animal \& Plant Sciences 25(1) : 3799-3813.

Morou B, 2010. Impact de l'occupation des sols sur I'habitat de la girafe au Niger et enjeux pour la sauvegarde du dernier troupeau de girafes de l'Afrique de l'Ouest. Mémoire de thèse de doctorat en Biologie appliquée, Faculté des Sciences et Techniques, Université Abdou Moumouni de Niamey. 198p

Muntz MA, Faure ML, Laine ME, 1905. Etudes sur la perméabilité des terres faites en vue de l'arrosage. France Min. de l'Agr. Dir. Gén. des Eaux et Forêts, Ann. Forêts-Hydraulique, $33: 45-96$

Noma AS, 2017. Evaluation du comportement de l'Eucalyptus camaldulensis dans les demilunes et banquettes forestières du plateau de Satara (commune rurale de Simiri, région de Tillabéry sud-ouest Niger). Mémoire de Master en Sciences de l'Environnement, Faculté des Sciences et Techniques, Université Abdou Moumouni de Niamey, $27 \mathrm{p}$

Oumarou M, 2003. Etudes écologique, floristique, phytogéographique et phytosociologique des inselbergs du Bénin. Thèse de doctorat, Laboratoire de Botanique Systématique, Faculté des Sciences, Université Libre de Bruxelles, 210p.

Raunkiaer C, 1934. The use of leaf size in biological plant geography (pp 368-378, I pl., 5 tabl.) In : The life forms of plants and statistical plant geography. Clarendon press, Oxford, $632 \mathrm{pp}$.

Roose E, Blanc PA.A, De Freitas PL, 1993.Un simple test de terrain pour évaluer la capacité d'infiltration et le comportement hydrodynamique des horizons pédologiques superficiels: méthode et exemples, Cah. Orstom, sér. P\&OL, V. XXVIII, 2 : 413-419

Saadou M, 1990. La végétation des milieux drainés nigériens à l'Est du fleuve Niger. Thèse de Docteur ès - Sciences Naturelles, Université de Niamey. 395 p. + annexes.

SE/CNEDD, 2006. Programme d'action national pour l'adaptation aux changements Climatiques. Rapport d'études, Cabinet du Premier Ministre, République du Niger, $79 \mathrm{p}$.

Sinsin B, 1993: Phytosociologie, écologie, valeur pastorale, production et capacité de charge des pâturages naturels du périmètre NikkiKalalé au Nord-Bénin. Thèse de Doctorat en
Sciences Agronomiques. Université Libre de Bruxelles, Belgique. 390 pp.

Sinsin B, 2001. Formes de vie et diversité spécifique des associations des forêts claires du nord du Benin. Syst. Geogr. Pl. 71 : 873-888.

Souley YK, 2008.L'évolution de l'occupation des sols à l'échelle des bassins versants de Wankama et Tondi Kiboro : Conséquences sur les débits. Mémoire de DEA en Aménagement et Gestion des Ressources Naturelles, Faculté des Lettres et Sciences Humaines, Département de Géographie, Université Abdou Moumouni de Niamey, $80 \mathrm{p}$.

Soumana I, 2012. Outil d'analyse des spectres phytogéographiques des pâturages sahéliens. Fiche technique $n^{\circ} 2$, INRAN, $3 p$.

SP/SDR, 2010. Stratégie de Développement Rural au Niger. Plan d'Action, Rapport d'études, Secrétariat Permanent, Cabinet du Premier Ministre, République du Niger

Taïbi AN, 2015. Désertification et dégradation : Réinterrogation des concepts à la lumière d'exemples africains. Thèse de doctorat en Géographie, Université d'Angers, $170 \mathrm{p}$.

Walkley AJand Black IA, 1934. Estimation of soil organic carbon by the chromic acid titration method. Soil Sci. 37: 29-38

Yacouba AS, Yadji G, Tchicama MM, Issa NA, 2018. Caractérisation des sols de Guillé Koira et Lassourou dans les communes rurales d'Imanan et Tondikandia au Niger pour une meilleure culture de riz autour des mares. Int. J. Biol. Chem. Sci. 12(6) : 2474-2485

Youssifi S, 2014. Caractérisation des peuplements ligneux des agrosystèmes d'Aguié et potentialité de séquestration du carbone de Faidherbia albida (Del.). Achev. Mémoire de Master en Biodiversité et Gestion de l'Environnement Soudano-Sahélo-Saharien, Université Dan Dicko Dankoulodo de Maradi, Niger, 47 p. 